\title{
A COMPARATIVE FLUORESCENCE POLARIZATION STUDY OF $c i s-$ PARINAROYLPHOSPHATIDYLCHOLINE AND DIPHENYLHEXATRIENE IN MEMBRANES CONTAINING DIFFERENT AMOUNTS OF CHOLESTEROL
}

\author{
A. CHRISTIANSSON*, F.A. KUYPERS, B. ROELOFSEN, K.W.A. WIRTZ and J.A.F. OP \\ DEN KAMP** \\ Department of Biochemistry, State University of Utrecht, University Center "De Uithof", \\ Padualaan 8, NL.3584 CH Utrecht (The Netherlands)
}

Received March 13th, 1984 accepted May I0th, 1984

The steady state fluorescence anisotropy $\left(r_{s}\right)$ of 1 -acyl-2 -cis parinaroyl phosphatidylcholine (PnPC) was compared with that of diphenylhexatriene (DPH) in a variety of model- and biological membrane systems. The fluorescence anisotropy of both probes responded similarly to temperature changes and variations in the acyl chain composition in phosphatidylcholine (PC) liposomes. The presence of proteins and cholesterol increased $r_{s}$ for both DPH and PnPC in the biological membranes as compared to the isolated polar membrane lipids. Comparison of DPH and PnPC in dipalmitoyl-PC-liposomes with and without $50 \mathrm{~mol} \%$ cholesterol, showed at temperatures above the phase transition of pure dipalmitoyl-PC the presence of cholesterol increased the $r_{s}$-value for DPH strongly, whereas the $r_{s}$-value for PnPC was much less affected. In the cholesterol-sich erythrocyte membrane as well as in microsomes from Morris hepatoma 7787, which have an increased cholesterol content as compared to normal rat liver microsomes, the $r_{s}$ of DPH was higher than that of PnPC. No large differences between the $r_{s}$-values of both probes were evident in the normal cholesterol-poor rat liver microsomes. These effects are discussed in terms of structural differences between the probes and variation of cholesterol content. Alterations in the fatty acid composition of PC present in human erythrocyte membranes were introduced with the aid of a PC-specific transfer protein. Fluorescence anisotropy values of both probes hardly changed upon enrichment of the red cell membrane with either dipalmitoyl PC or 1-palmitoyl-2-arachidonyl PC.

Keywords: parinaroyl phosphatidylcholine; diphenylhexatriene; erythrocyte membrane; cholesterol; PC-transfer protein.

\section{Introduction}

The fluorescent fatty acid cis-parinaric acid $(9,11,13,15$-cis, trans, trans, cisoctadecatetraenoic acid) and its all-trans isomer are spectroscopically well-characterized probes [1]. They have been used in lipid model systems and biological mem. branes to study lipid-lipid and lipid-protein interactions [2-6]. A number of

\footnotetext{
* Present address: Department of Microbiology, University of Lund, Sölvegatan 21, S-22362 Lund, Sweden.

**To whom correspondence should be sent.
}

0009-3084/84/\$03.00 1984 Elsevier Scientific Publishers Ireland Ltd.

Published and Printed in Ireland 
different phospholipid analogues of parinaric acid have been synthesized recently and their partitioning behaviour between solid and fluid lipid phases in liposomes is known $[7,8]$. Since parinaric acid is a naturally occurring fatty acid, parinaroyl phospholipids are likely to behave as almost perfect lipid probes. The probe molecule can be expected to cause minimal disturbance of the lipid bilayer since the conjugated polyene chromophore does not differ much from a normal monounsaturated acyl chain with respect to conformation [9]. The application of parinaric acid containing phospholipid as fluorescent probe in biological membranes has been very limited [10] mainly because intact phospholipids are difficult to insert into biological membranes. In the present study we have overcome this problem using a PC-specific transfer protein which enabled us to introduce PnPC in erythrocyte and microsomal membranes.

In order to explore the usefulness of PnPC as a probe for lipid-lipid and lipidprotein interactions its fluorescent properties were compared with those of the more widely used probe, DPH [11]. As a first approach the measurement of the steady-state fluorescence anisotropy $\left(r_{s}\right)$ was carried out. It has been shown that in case of DPH [12-15], parinaric acid and PnPC [16] anisotropy values are determined by two components: The residual anisotropy $\left(r_{\infty}\right)$ which is related to the order parameter of the probe and a dynamic term $\left(r_{f}\right)$ which depends upon the apparent rotational correlation time and the fluorescence life time of the probe [17]. The relation between these factors has been discussed in detail by van Blitterswijk et al. [18]. They showed that when the $r_{s}$-values of DPH in a membrane exceed a value of 0.13 the contribution of the dynamic term to the steady-state fluorescence anisotropy is very small. In this case the largest contribution comes from the $r_{\infty}$-value which means that $r_{s}$ gives mainly structural information about the probe and its surrounding [18]. Many eukaryotic membranes contain cholesterol which strongly modifies the physical behaviour of the membrane lipids. Time-resolved measurements have revealed for both DPH and parinaric acid that $r_{\infty}$ is the term mainly affected by cholesterol in fluid phase lipids [16.18]. With this information in mind we have compared the $r_{s}$-values for PnPC and DPH in PC-containing liposomes in the presence and absence of cholesterol. In addition, DPH and PnPC have been introduced into biological membranes with various cholesterol contents. Finally we have attempted to determine to what extent $r_{s}$ measurements of PnPC and DPH are sensitive enough to monitor small changes in the fatty acid composition of the phospholipids in the erythrocyte membrane [19].

\section{Materials and Methods}

\section{Lipids}

Dipalmitoyl-PC and dimyristoyl-PC were gifts from colleagues at the department and were synthesized following established procedures [20] 1-Palmitoyl-2-arachidonoyl-PC was obtained from Avanti Polar Lipids Inc., (Birmingham, AL), egg PC from Sigma (St. Louis, MO) and cis-parinaric acid was purchased from Moleculas 
Probes (Plano, TX). 1-Acyl lyso-PC was prepared from egg PC by phospholipase $\mathrm{A}_{2}$ treatment and 1-acyl-2-parinaroyl-PC ( $\mathrm{PnPC}$ ) was synthesized as described earlier [21]. The purified PnPC was stored at a concentration of $4 \mu \mathrm{M}$ in $95 \%$ ethanol (Merck, fluorescence grade) under an argon atmosphere. 1 mol\% butylated hydroxytoluene was added as antioxidant and the lipid was stored at $-20^{\circ} \mathrm{C}$ in the dark. DPH was obtained from Koch-Light (Colnbrook, U.K.) and was stored at a concentration of $2 \mathrm{mM}$ in tetrahydrofuran in the dark.

\section{Membranes}

Erythrocytes were isolated from freshly drawn human blood collected into standard acid/citrate/dextrose buffer [22]. The blood was centrifuged for $5 \mathrm{~min}$ at $2500 \times \mathrm{g}$. After removal of the supernatant and the buffy coat by aspiration, the cells were washed three times in cold $\left(4^{\circ} \mathrm{C}\right)$ phosphate-buffered saline $(150 \mathrm{mM}$ $\mathrm{NaCl}, 5 \mathrm{mM}$ sodium phosphate, $\mathrm{pH} 8.0$ ). Sealed erythrocyte ghosts were prepared essentially as described by Steck [23] and were stored in phosphate buffered saline at a concentration corresponding to $2.5 \mu \mathrm{mol}$ phospholipid phosphorus per $\mathrm{ml}$. The sealed ghosts contained $<4 \%$ of the original concentration of hemoglobin. The degree of sealing of the ghosts was determined by measuring the activity of glyceraldehyde 3-phosphate dehydrogenase (EC 1.2.1.12), which is associated with the cytoplasmic side of the membrane. The activity of the ghost preparation was compared to that of Triton X-100 solubilized ghosts [24]. Approximately $95 \%$ of the ghosts were sealed with a correct right side-out orientation of the membrane. The sealed ghosts were stored at $4^{\circ} \mathrm{C}$ and were used within 2 days after preparation. Microsomes were prepared as described previously [25] from normal rat liver and Morris hepatomas 7787.

\section{Preparation of lipid extracts}

Sealed ghosts were extracted as described by Rose and Oklander [26] and the microsomes were extracted according to Bligh and Dyer [27]. After evaporation of the solvent the extracts were redissolved in chloroform/methanol $(2: 1, \mathrm{v} / \mathrm{v})$ and stored under $\mathrm{N}_{2}$ at $-20^{\circ} \mathrm{C}$. Part of the extracts were used for fluorescence measurements of total lipid extracts. A second part was applied on silica gel plates and the neutral lipids including cholesterol were separated from the phospholipids using the solvent system ether/hexane $(85: 15, \mathrm{v} / \mathrm{v})$. The phospholipid fraction was recovered and used for fluorescence measurements. The phospholipid content of the extracts was determined according to Rouser [28]. Cholesterol [29] and membrane protein content [30] were determined following established procedures.

\section{Phosphatidylcholine transfer protein}

The PC-transfer protein was purified from bovine liver as described before [31]. The protein was stored in $50 \%$ glycerol at $-20^{\circ} \mathrm{C}$.

\section{Modification of erythrocyte PC in ghosts}

Unilamellar donor vesicles containing the required phosphatidylcholine plus 
cholesterol and phosphatidic acid at a molar ratio of 47:47:6 were prepared by sonication at a temperature above the phase transition of the PC as described earlier [19].

The incubation mixture consisted of sealed ghosts (approx. $1 \mu \mathrm{mol}$ phospholipid! $\mathrm{ml}), \mathrm{PC}$ transfer protein $(2 \mu \mathrm{M})$ and donor vesicles corresponding to a molar ratio of vesicle PC/erythrocyte PC of $2: 1$. The mixture was incubated at $37^{\circ} \mathrm{C}$ in a clinical blood rotator at $4 \mathrm{rev} / \mathrm{min}$. The samples were incubated for $2 \mathrm{~h}$. 1-Palmitoyl-2arachidonoyl-PC was introduced in a similar manner into intact red blood cells as described [19]. After the exchange the cells were lysed according to Dodge [32]. The membranes were washed and resuspended in phosphate buffered saline and used for fluorescence measurements. No differences in $r_{s}$ were found for the same type of membranes labelled as sealed ghosts or as Dodge ghosts. The extent of PC replacement in the erythrocyte membranes are determined as described in detail elsewhere [19].

\section{Preparation of samples for fluorescence measurements}

(a) Multilamellar liposomes. For labelling with PnPC, lipid extracts containing 100-150 nmol phospholipid were added to screwcapped glass tubes and the tubes were filled with $\mathrm{N}_{2}$. As an antioxidant butylated hydroxytoluene (BHT) was added $(0.3 \mathrm{nmol})$ from a stock solution in ethanol (spectroscopic grade). Finally 2- 3 nmol of PnPC was added to the mixtures. The solvent was evaporated under $\mathrm{N}_{2}$ and the lipid mixtures were kept in the dark. Residual traces of solvent were removed under vacuum. The lipids were rehydrated by addition of $2 \mathrm{ml}$ of phosphate buffered saline and dispersed by vortexing. Fluorescence blanks were prepared in exactly the same manner, but the PnPC was omitted. For DPH-labelling the $2 \mathrm{mM}$ stock solution of DPH in tetrahydrofuran was diluted 2000-fold into phosphate buffered saline by blowing the stock solution into a phosphate buffered saline solution with rapid agitation. The tetrahydrofuran was removed with a stream of $\mathrm{N}_{2}$. The liposomes were prepared as described above except that the BHT was omitted and that the lipid concentration was twice that used for PnPC-labelling. To $1 \mathrm{ml}$ of liposomes, $1 \mathrm{ml}$ of the diluted DPH-solution was added and the mixture was incubated for one hour at $37^{\circ} \mathrm{C}$. The blank consisted of $1 \mathrm{ml}$ of liposomes diluted with $1 \mathrm{ml}$ of phosphate buffered saline.

(b) Membranes. Erythrocyte ghosts corresponding to $180 \mathrm{nmol}$ phospholipid were added to $4 \mathrm{ml}$ of phosphate buffered saline, which was previously llushed with argon in a screwcapped test tube. BHT $(0.4 \mathrm{nmol})$ was added and the mixture was divided in two parts. To the fluorescence sample 3-4 nmol PnPC was added by rapid injection of an ethanol solution into the incubation mixture. This procedure leads to the formation of unilamellar phospholipid vesicles [33]. To the blank, an amount of ethanol corresponding to the PnPC addition was added. PC transfer protein (final concentration $0.08--0.16 \mu \mathrm{M}$ ) was added to both sample and blank. The tubes were flushed with argon, closed and incubated at $25^{\circ} \mathrm{C}$ for $1.5-2 \mathrm{~h}$. Care was taken to protect the tubes from light. The microsomes were labelled in a 
similar manner except that the microsomes were used at a concentration of $0.1 \mathrm{mg}$ membrane protein $/ \mathrm{ml}$ and that $4-8 \mathrm{nmol}$ PnPC was added. DPH-labelling was performed with membranes in the same way as described for labelling of multilamellar liposomes. Membranes at the same concentrations as that used for PnPC. labelling were diluted with an equal volume of DPH solution and were incubated for $1 \mathrm{~h}$.

\section{Fluorescence measurements}

The incorporation of DPH and PnPC into membranes was monitored by measurement of the fluorescence increase with a Perkin-Elmer MPF-3 spectrofluorometer. The samples were incubated in a thermostated cuvette holder. To avoid local bleaching in the excitation beam, the samples were stirred continuously with a small magnetic bar in the cuvette [21]. For PnPC-containing samples the excitation wavelength was $324 \mathrm{~nm}$ (slit $2 \mathrm{~nm}$ ) and the emission wavelength was $420 \mathrm{~nm}$ (slit $40 \mathrm{~nm}$ ). To eliminate quenching caused by probe-probe interaction, the final PnPC to lipid ratio was maintained at a value of $1 / 100$ or less in all samples. DPH was excited at $365 \mathrm{~nm}$ (slit $8 \mathrm{~nm}$ ) and the emission was observed at $420 \mathrm{~nm}$ (slit $8 \mathrm{~nm}$ ). To reduce scattering contributions to the fluorescence signal the MPF3 $390 \mathrm{~nm}$ cut-off filter was placed in the emission beam. Fluorescence polarization was measured in a Hübweiz Flupol T-format apparatus $[34,35]$. The steady state fluorescence anisotropy $\left(r_{s}\right)$ and polarization $(P)$ are determined from the emission intensities through an analyzer oriented parallel $\left(J_{\|}\right)$and perpendicular $\left(I_{\perp}\right)$ to the direction of polarization of the excitation light. The background intensities were subtracted from $I_{\|}$and $I_{\perp}$ before calculations were performed. Using the equations given in (18), the fluorescence polarization was calculated as defined in the equation

$$
r_{s}=\frac{I_{\|}-I_{1}}{I_{\|}+2 I_{1}}
$$

\section{Results and Discussion}

\section{Dipalmitoyl PC liposomes}

The temperature dependence of the fluorescence anisotropy of PnPC and DPH was compared in dipalmitoyl PC-liposomes (Fig. 1). The concentration of lipid was such that no scattering depolarization was evident, as judged by a constant $r_{s}$ upon dilution of the samples. Both probes exhibited a rapid decrease of the $r_{s}$ at the phase transition of dipalmitoyl PC at approx. $39-42^{\circ} \mathrm{C}$. In the gel phase the $r_{s}$ of DPH was slightly higher than that of PnPC. The $r_{s}$ for DPH in dipalmitoyl PC agrees well with previously published results [17]. The phase transition of dimyristoyl PC was similarly monitored by both probes around $23^{\circ} \mathrm{C}$ (data not shown). When $50 \mathrm{~mol} \%$ cholesterol was included in dipalmitoyl PC liposomes, the phase transition was abolished (Fig. 1). The anisotropy of the two probes was quite different in the presence of cholesterol. For DPH the level of the $r_{s}$ through- 
out the temperature interval measured was lower than the $r_{s}$ found in the gel phase of pure DPPC. This is in agreement with earlier observations [17]. With decreasing cholesterol content the temperature dependence of the $r_{s}$ successively approached the curve for pure dipalmitoyl PC (not shown and [17]). For PnPC embedded in liposomes containing equal amounts of dipalmitoyl PC and cholesterol the level of the $r_{s}$ was approximately mid-way between the levels in pure dipalmitoyl PC liposomes immediately before and after the phase transition (Fig. 1). The results suggest that the $r_{s}$ of DPH is more strongly affected by the presence of cholesterol in the liquid crystalline phase than is the $r_{s}$ of PnPC. and that the reverse holds tor the gel phase. Similar results are obtained with dimyristoyl PC and egg PC (data not shown).

\section{Microsomal membranes}

DPH is easily incorporated into biological membranes upon addition of DPH to the aqueous phase. Due to the strongly increased quantum yield in lipid environments compared to aqueous environments the incorporation can be monitored by the increase in fluorescence intensity upon incorporation [11]. The transfer of PnPC between phospholipid vesicles has been shown to be facilitated by the PC transfer protein [21].

The same protein was used to transfer the probe into erythrocytes and microsomes. Figure 2 shows a typical example of the incorporation procedure. The injection of $\mathrm{PnPC}$ vesicles, in which fluorescence is quenched, due to probe-probe

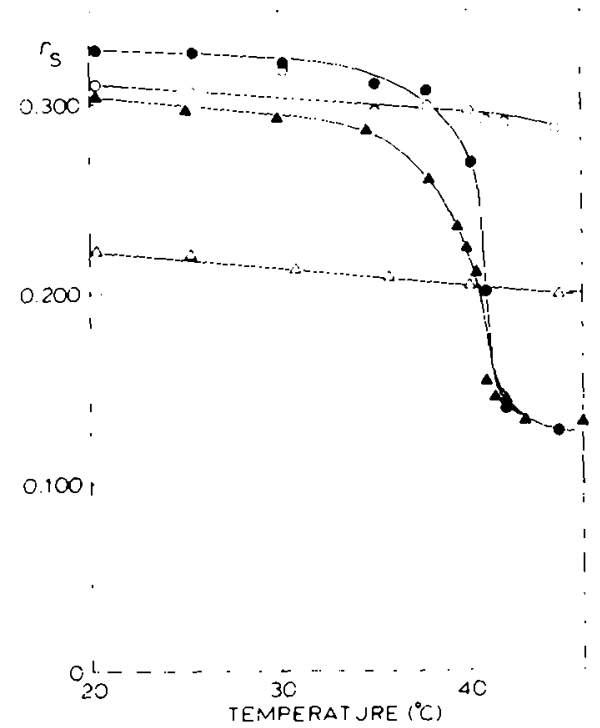

Fig. 1. Effect of temperature on the steady-state fluorescence anisotropy, $r_{s}$, of PnPC (4. $\left.\wedge\right)$ and DPH $(\bullet, 0)$ embedded in DPPC liposomes (filled symbols) or liposomes consisting of DPPC and $50 \mathrm{~mol} \%$ cholesterol (open symbols). See Materials and Methods for sample preparation. 


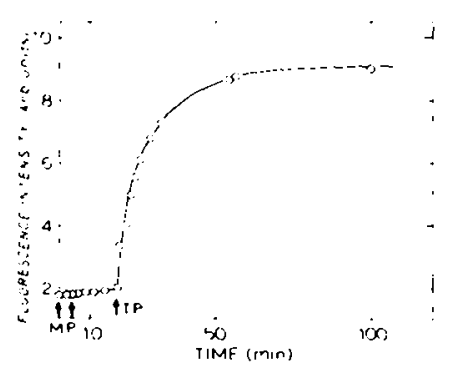

Fig. 2. Incorporation of PnPC into rat liver microsomes with the aid of phosphatidylcholine specific transfer protein. The time dependence of the fluorescence intensity was followed upon incubation of rat liver microsomes together with donor vesicles consisting of PnPC. The vesicles were produced by ethanol injection. Arrow $M$ : addition of microsomes (approx. $0.1 \mathrm{mg} \mathrm{mem}$ brane protein $/ \mathrm{ml}$ in buffer), arrow P: injection of PnPC. Arrow TP: addition of phosphatidylcholine specific transfer protein. The incubation was performed at $25^{\circ} \mathrm{C}$.

interactions (arrow P) into a cuvette containing rat liver microsomes resulted in a very slow fluorescence increase. This indicates that the spontaneous transfer of PnPC from the donor vesicles to the membranes was very slow. Upon addition of the transfer protein (arrow TP) there was a rapid increase in fluorescence, indicating a transfer of PnPC to the membranes. After the initial increase, the fluorescence intensity gradually levelled off and approached a plateau value. The final level of fluorescence depended upon the amount of PnPC present within the donor vesicles. Since the transfer protein facilitates a 1:1 exchange of PC between donor and acceptor membranes, the final equilibrium situation will depend upon the amount of PC available in the donor and acceptor membranes. Similar incorporation curves as shown in Fig. 2 were obtained for erythrocyte membranes and microsomes obtained from Morris hepatoma 7787 (not shown).

The temperature dependence of the $r_{s}$ of PnPC was compared to that of DPH in the different membranes. In order to compare the influence of protein and cholesterol on the anisotropy of the two probes, we also determined the $r_{s}$ of the two probes embedded in liposomes made from the total lipid extracts and from cholesterol depleted lipid extracts. Figure 3 illustrates the results for rat liver microsomes and microsomes from Morris hepatoma 7787. Rat liver microsomes have a low cholesterol content (cholesterol/phospholipid molar ratio 0.07) whereas the cholesterol content in 7787 microsomes is higher (ratio 0.21 ). Furthermore 7787 microsomes contain more protein than normal rat liver microsomes [36]. The concentration of both microsomal preparations used for the PnPC fluorescence measurements equaled $100-130 \mathrm{nmol}$ of phospholipid in a volume of $2 \mathrm{ml}$ and resulted in an absorbance value at $420 \mathrm{~nm}$ of 0.15 . For DPH measurements the samples were diluted twice during the labelling procedure. Due to limitations in instrumental sensitivity it was not possible to dilute the samples further without loss of accuracy in readings for PnPC labeled samples. Hence, all microsome samples were subject to some scattering depolarization. Properly diluted samples would 


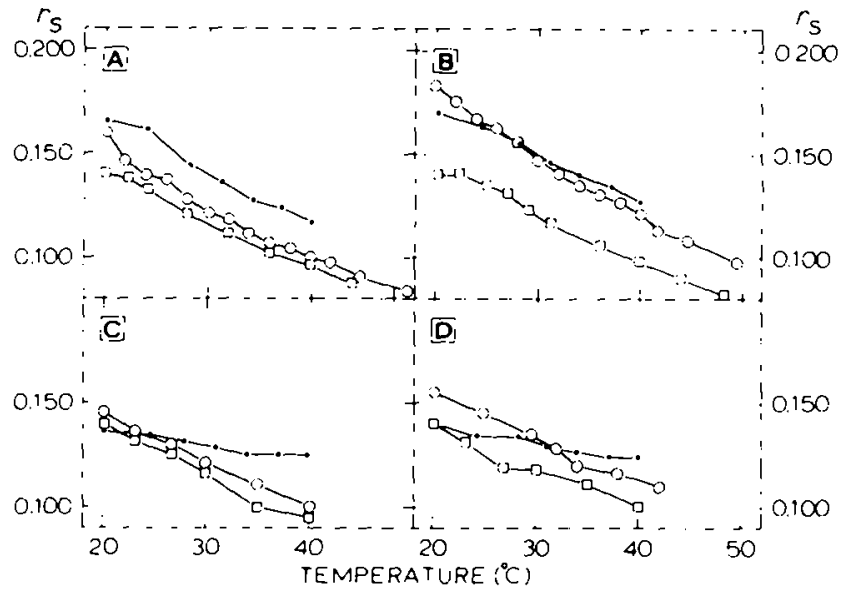

Fig. 3. The effect of membrane proteins and cholesterol on the thuorescence anisotropy of $\mathrm{PnPC}$ and DPH in normal rat liver microsomes $(\mathrm{A}, \mathrm{C})$ and in microsomes from Morris hepatoma 7787 (B,D). Intact membranes (-.-), total lipid extracts $(-(-)$ ) and cholesterol depleted lipid extracts $(-\neg-$.$) were labelled with DPH (A and B) or PnPC (C and D) as described in Materials$ and Methods.

have given higher $r_{s}$-values. Nevertheless, some useful conclusions can be drawn. Firstly, no lipid transitions are seen in the temperature interval studied, neither for the membranes nor for the lipid extracts. Secondly, the $r_{s}$ for DPH in 7787 microsomes was consistently higher than in liver microsomes and the $r_{s}$ in the membranes was higher than in the lipid extracts (Fig. 3A,B). Furthermore, the difference in $r_{s}$ between total lipid extracts and lipid extracts without cholesterol was very small for the normal liver extracts but larger for 7787 extracts. In agreement with earlier observations $[11,15,17,37]$, our results suggest that both proteins and cholesterol increase the anistropy of DPH in biological membranes when compared with the model membranes. The fact that the anisotropy difference between the total lipids and the cholesterol free lipid extracts was larger in extracts from 7787 microsomes than in extracts from normal rat liver microsomes can be explained by the higher cholesterol content of 7787 microsomes. The corresponding curves for PnPC labelled microsomes are shown in Figs. 3C and 3D. The fluorescence anistropy for PnPC in the two membranes did not differ significantly. This is probably due to a difference in the scattering depolarization of the two samples. Attempts to extrapolate the fluorescence anisotropy to zero absorbance by dilution of the samples [38], although subject to large uncertainty, indicated that PnPC had a higher $r_{s}$ in 7787 microsomes than in normal microsomes (not shown). In the lipid extracts the same relative differences in anisotropy were found as shown for DPH (Figs. 3C and 3D). Thus, the $r_{s}$ of PnPC is influenced both by protein and cholesterol in biological membranes in a qualitatively similar manner to that of DPH. Note that the $r_{s}$-curves for both probes embedded in total lipids and cholesterol- 
depleted lipids are similar for liver microsomes and also for the cholesterol-depleted extract of 7787. However, the temperature dependence of PnPC in the total lipid extract of 7787 microsomes exhibited lower $r_{s}$-values than that of DPH. The observations made in the dipalmitoyl PC liposome system (Fig. 1) that DPH is more sensitive than PnPC to the presence of cholesterol in lipid membranes appears to hold in these extracts too. The difference in $r_{s}$ between DPH and PnPC in these lipid extracts is however smaller than in Fig. 1 . This is to be expected, since the cholesterol content of these membranes is lower than $50 \mathrm{~mol} \%$.

\section{Erythrocytes}

In Fig. 4 a comparison is made of the behaviour of DPH and PnPC in human erythrocyte membranes. The cholesterol/phospholipid ratio in this membrane is $0.8-0.9$ and thus considerably higher than in the microsomes. The steady-state fluorescence anisotropy for DPH in the membranes was much higher in the microsomes (Figs. 4A and 3A, respectively), probably as a result of the high cholesterol content in the red cell membrane. The data are in good agreement with the steady. state data of Kinosita et al. [39]. DPH in liposomes made from total erythrocyte lipids had a slightly lower anisotropy than in the ghosts, in agreement with earlier studies [40]. In the cholesterol-depleted lipids, the $r_{s}$ was much lower, which confirms that cholesterol was the cause of the high $r_{s}$ in the erythrocyte membrane. The fluorescence anistropy for PnPC in erythrocyte membranes (Fig. 4B) was markedly lower than that for DPH. This lower value was not due to scattering depolarization, as was the case for the microsomes, since dilution of the samples still yielded the same $r_{s}$. The absorbance of the samples at $420 \mathrm{nmol}$ was about 0.15 ; however, a substantial part of this was due to the presence of hemoglobin. The difference in $r_{s}$ between membranes and total lipid extracts was (probably) insignificant (Fig. 4). The $r_{s}$ in lipids without cholesterol was lower than that in

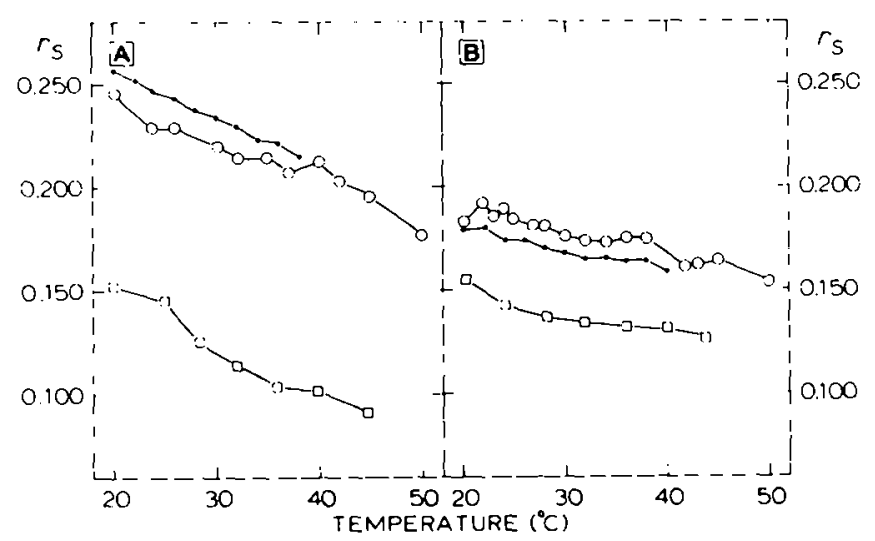

Fig. 4. The effect of membrane proteins and cholesterol content on the fluorescence anisotropy of DPH (A) and PnPC (B) in sealed human erythrocyte ghosts. - - , ghosts; - - , total lipids; $-\square-$, cholesterol depleted lipids. 
the total lipids, although the difference was much smaller than in the case of DPH. Once again, the differences in $r_{s}$ of the two probes in the presence and absence of cholesterol agrees with their behaviour in model systems (Fig. 1).

It is possible that the different anisotropy values which have been observed for $\mathrm{DPH}$ and PnPC in the presence of cholesterol can be ascribed to differences in probe structure and probe localization. The thickness of the DPH molecule approximates that of an acyl chain and its ordering in a membrane is presumed to reflect the order of the acyl chains [17], be it that the precise localization of the probe is unknown. The parinaroyl chromophore is relatively rigid (carbon atoms 9-16) and its movement will depend not only upon the movement and order of the surrounding acyl chains but also on depolarizing motions of the acyl chain in which it is present [16]. Cholesterol increases mainly the order of the first 8-10 carbon atoms of the phospholipid acyl chains [41]. This interaction apparently affects PnPC in a different way than DPH, presumably because the latter probe is not fixed in a defined position in the bilayer. However, since differences in fluorescence lifetime and rotational correlation time also affect the fluorescence anisotropy values, lifetime measurements and time-resolved anisotropy measurements are necessary to clearly establish the nature of the differences between the two probes.

The effect of modification of the acyl composition of the human erythrocyte ghosts was investigated with the aid of PC transfer protein. Native erythrocyte PC was replaced by different PC species with defined acyl chain composition. In Fig. 5 the temperature dependencies of the $r_{s}$ of DPH and PnPC are compared in normal and dipalmitoyl PC-modified membranes. In this particular experiment $16 \%$ of the native PC was replaced by dipalmitoyl PC. This amounts to approx. 5\% of the total phospholipids in the membrane. Both DPH and PnPC monitored the effect of the PC-exchange as a very small increase in $r_{s}$. Similar results as in Fig. 5 were obtained after replacing $23 \%$ of the erythrocyte PC with dimyristoyl PC. Replace-

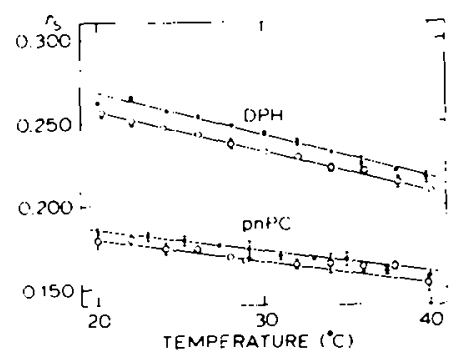

Fig. 5. Comparison between the temperature dependence of the fluorescence anisotropy of DPH (upper two curves) and PnPC (lower two curves) in normal (-0-) and DPPC-modified (--) sealed erythrocyte ghosts. Sealed ghosts were modified by incubating them in the presence of donor vesicles consisting of DPPC/cholesterol/PA (47:47:6 mol\%) and PC-specific transfer protein as described in Materials and Methods. $16 \%$ (mol) of the erythrocyte PC was replaced by DPPC. The error bars denote S.D. of repeated measurements of the same sample. 
ment with 1-palmitoyl-2-arachidonoyl-PC to an extent of $30 \%$ did not result in anisotropy values significantly different from nonmodified membranes (data not shown). The small differences in $r_{s}$-values which are observed upon changes in fatty acid composition have been observed also in plasma membranes of murine leukemia EL4 cells after fatty acyl modifications [42]. In addition, it is noteworthy that also in liposomal membranes differences in fatty acyl constituents give rise to limited changes in $r_{s}$-values, particularly when unsaturated acyl chains are present [43]. On the other hand, limited changes in fatty acyl composition of the erythrocyte PC can induce drastic modifications in erythrocyte shape and general membrane properties, such as its permeability [19]. Therefore it can be concluded that steady-state fluorescence measurements are not sensitive enough to monitor subtle changes in acyl chain composition in cholesterol containing membranes.

\section{Acknowledgements}

This investigation was carried out under the auspices of The Netherlands Foundation for Chemical Research (S.O.N.) and with financial support from The Netherlands Organization for the Advancement of Pure Research (Z.W.O.) and the Koningin Wilhelmina Fonds for Cancer Research in the Netherlands. A.C. was supported by a post-doctoral fellowship from the Swedish Natural Science Research Council. Mrs. M. van Linde is gratefully acknowledged for the preparation of purified PC transfer protein and Drs. S. de Laat and P.T. van der Saag are acknowledged for their assistance with fluorescence measurements.

\section{References}

1 L.A. Sklar, B.S. Hudson, M. Petersen and J. Diamond, Biochemistry, 16 (1977) 813-819.

2 L.A. Sklar, B.A. Hudson and R.D. Simoni, Biochemistry, 16 (1977) 819-828.

3 L.A. Sklar, G.P. Miljanich and E.A. Dratz, Biochemistry, 18 (1979) 1707-1716.

4 D. Kimelman, E.S. Tecoma, B.K. Wolber, B.S. Hudson, W.T. Wickner and R.D. Simoni, Biochemistry, 18 (1979) 5874-5880.

5 F. Schroeder, Eur. J. Biochem., 132 (1983) 509-516.

6 D.A. Rintoul, S.-M. Chou and D.F. Silbert, J. Biol. Chem., 254 (1979) 10070-10077.

7 R. Welti and D.F. Silbert, Biochemistry, 21 (1982) 5685-5690.

8 R. Welti. Biochemistry, 21 (1982) 5690-5694.

9 L.A. Sklar, B.S. Hudson and R.D. Simoni, Proc. Natl. Acad. Sci. U.S.A., 72 (1975) 1649 1653.

10 E.L. Pugh, M. Kates and A.G. Szabo, Chem. Phys. Lipids, 30 (1982) 55-69.

11 M. Shinitzky and Y. Barenholz, Biochim. Biophys. Acta, 515 (1978) 367-394.

12 K. Kinosita, S. Kawato and A. Ikegami, Biophys. J., 20 (1977) 289- 305.

13 K. Hildenbrand and C. Nicolau, Biochim. Biophys. Acta, 553 (1979) 365-377.

14 I. Jähnig, Proc. Natl. Acad. Sci. U.S.A., 76 (1979) 6361-6365.

15 M.P. Heyn, FEBS Lett., 108 (1979) 359-364.

16 P.K. Wolber and B.S. Hudson, Biochemistry, 20 (1981) 2800-2810.

17 S. Kawato, K. Kinosita and A. Ikegami, Biochemistry, 17 (1978) 5026-5031.

18 W.J. van Blitterswijk, R.P. van Hoeven and B.W. van der Meer, Biochim. Biophys. Acta, 644 (1981) 323-332. 
19 F.A. Kuypers, B. Roelofsen, J.A.F. Op den Kamp and L.L.M. van Deenen, Biochim. Biophys. Acta, 769 (1984) 337-347.

20 L.L.M. van Deenen and G.H. de Haas, Adv. Lipid Res., 29 (1964) 168-234.

21 P. Somerharju, H. Brockerhoff and K.W.A. Wirtz, Biochim. Biophys. Acta, 649 (1981) 521- 528 .

22 H.M. Anderson and J.C. Turner, J. Clin. Invest., 39 (1960) $1-7$.

23 T.L. Steck, in: Ł. Korn (Ed.), Methods in Membrane Biology, Vol. 2, 1974, pp. 245 - 281.

24 T.L. Steck and J.A. Kant, Methods Enzymol., 31 (1974) 172-188.

25 G.P.M. van Heusden, T.P. van der Krift, K.Y. Hostetler and K.W.A. Wirtz, Cancer Res., 43 (1983) $4207 \cdot 4210$.

26 H.G. Rose and M. Oklander, J. Lipid Res., 6 (1965) 428-431.

27 F.G. Bligh and W.J. Dyer, Can. J. Biochem. Biophys., 37 (1959) $911-917$.

28 G. Rouser, S. Fleischer and A. Yamamoto, Lipids, 5 (1970) $494-496$.

29 W. Richmond, Clin. Chem., 19 (1973) 1350-1355.

30 O.H. Lowry, N.J. Rosebrough, A.L. Farr and R.J. Randall, J. Biol. Chem., 193 (1951) 265275.

31 J. Westerman, H.H. Kamp and K.W.A. Wirtz, Methods Enzymol., 98 (1983) $581-586$.

32 C.T. Dodge, C.D. Mitchell and D.J. Hanahan, Arch. Biochem. Biophys., 100 (1963) 119 -129.

33 S. Batzri and G.D. Korn, Biochim. Biophys. Acta, 298 (1973) 1015-1019.

34 S. de Laat, P.T. van der Saag and M. Shinitzky, Proc. Natl. Acad. Sci. U.S.A., 74 (1977) 4458.4461.

35 S. de Laat, P.T. van der Saaq, S.A. Nelemans and M. Shinitzky, Biochim. Biophys. Acta, 509 (1978) 188-193.

36 K.Y. Hostetler, B.D. Zermer and H.P. Morris, Cancer Res., 39 (1979) 2978 - 2983.

37 W.J. van Blitterswijk, P. Emmelot, H.A.M. Hilkmann, E.P.M. Oomen-Meulemans and M. Inbar, Biochim. Biophys. Acta, 467 (1977) $309-320$.

38 B.R. Lentz, B.M. Moore and D.A. Barrow, Biophys. J., 25 (1979) $489-494$.

39 K. Kinosita, R. Katooka, Y. Kimura, A. Gotoh and A. Ikegami, Biochemistry, 20 (1981) 4270.4277.

40 B. Aloni, M. Shinitzky and A. Livine, Biochim. Biophys. Acta, 348 (1974) 438-441.

41 J.H. Davis, M. Bloom, K.W. Butler and I.C.P. Smith, Biochim. Biophys. Acta. 597 (1980) 477.491 .

42 E. McVey, J. Yguerabide, D.C. Hanson and W.R. Clark, Biochim. Biophys. Acta, 642 (1981) $106-118$.

43 C.D. Stubbs, T. Kouyama, K. Kinosita and A. Ikegami, Biochemistry, 20 (1981) 4257 . 4262 . 